\title{
Fluoride Leachate Profile of Silver Diamine Fluoride
}

ISSN: 2637-7764

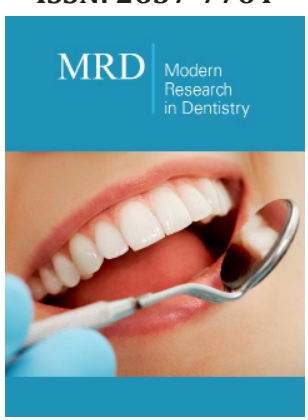

*Corresponding author: Jilen Patel, UWA Dental School, 17 Monash Avenue, Neelands WA 6009, Australia

Submission: 監 May 30, 2019

Published: 㘹June 06, 2019

Volume 4 - Issue 2

How to cite this article: Jilen $\mathrm{P}$, Robert $\mathrm{P}$ A, Nigel M K. Fluoride Leachate Profile of Silver Diamine Fluoride. Mod Res Dent. 4(2). MRD.000581.2019.

DOI: 10.31031/MRD.2019.04.000581

Copyright@ Jilen Patel, This article is distributed under the terms of the Creative Commons Attribution 4.0 International License, which permits unrestricted use and redistribution provided that the original author and source are credited.

\author{
Jilen Patel ${ }^{1 *}$, Robert P Anthonappa ${ }^{2}$ and Nigel M King ${ }^{3}$ \\ ${ }^{1}$ Paediatric Dental Registrar, Australia \\ ${ }^{2}$ Paediatric Dentistry, Australia \\ ${ }^{3}$ Professor Paediatric Dentistry, Australia
}

\section{Abstract}

Purpose: There has been a renewed interest in the use of silver diamine fluoride (SDF) in arresting caries. However, the high fluoride concentration has raised concerns when used in young children. Therefore, this study investigated the fluoride leachate profiles of SDF, $5 \%$ sodium fluoride varnish (NaF), SDF with Potassium Iodide (SDF+KI), and fluoridated toothpaste (FTP).

Method: Sixty enamel specimens were randomly grouped to receive topical application of NaF, SDF SDF+KI or FTP. Samples from each group were immersed into either neutral, basic or acidic artificial saliva solutions. Cumulative fluoride release and fluoride leachate profiles were measured at $24,48,96$, and 168 hours post-application using a fluoride ion selective electrode. The data was analysed using oneway ANOVA and Tukey tests.

Result: (i) Specimens in the NaF group exhibited a significantly higher fluoride leachate compared to $\mathrm{SDF}, \mathrm{SDF}+\mathrm{KI}$ and FTP groups $(\mathrm{p}<0.01)$, (ii) fluoride leachate profiles varied among the groups depending on the $\mathrm{pH}$ of the artificial saliva, iii) SDF showed a higher cumulative fluoride release compared to $\mathrm{NaF}$ (iv) $\mathrm{KI}$ application resulted in a lower measurable fluoride.

Conclusion: NaF exhibits a more sustained fluoride leachate profile compared with SDF. The application of KI following SDF appears to reduce the total measurable soluble fluoride.

Keywords: Fluoride; Silver diamine fluoride; Concentration; Leachate

\section{Introduction}

Recently, there has been a renewed interest in the use of silver diamine fluoride (SDF) in the management of caries especially in the high-risk groups. Clinical trials have shown SDF to be an easy to use, effective and safe topical agent with no significant adverse effects [1-4]. As a result, SDF is positioned as an efficient and equitable caries control agent that can be used to help meet the WHO Millenium oral health goals and fulfil the US Institute of Medicine's criteria

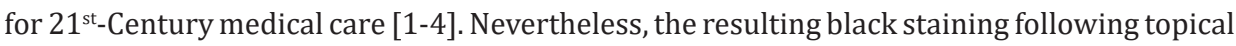
application of SDF, as well as the fluoride concentration, potential toxicity and chemical stability of silver fluoride compounds have all been commonly reported as barriers for SDF use [1,5-7]. In an attempt to overcome the unsightly black stain, use of potassium iodide (KI) immediately following SDF application has been advocated [8]. However, to date, there is limited literature investigating the fluoride release from SDF, and to our knowledge, there are no reports investigating the effect of KI application on fluoride release [9]. Therefore, this study sought to compare the fluoride leachate profiles of 38\% SDF, 38\% SDF with Potassium Iodide (SDF+KI), $5 \%$ sodium fluoride varnish $(\mathrm{NaF})$ and $0.32 \%$ fluoridated toothpaste (FTP). Furthermore, as fluoride release has been shown to vary depending on $\mathrm{pH}$, a secondary objective of this study was to evaluate the fluoride leachate profiles of the aforementioned agents in neutral, acidic and basic artificial saliva to better reflect the oral environment.

\section{Methods}

\section{Experimental groups}

Following ethical approval (Human Research Ethics Committee, University of Western Australia) and consent from the participants, human premolars $(n=30)$ with clinically sound enamel, which were extracted for orthodontic reasons were decoronated and sectioned bucco-lingually to produce a total of 60 enamel slabs. A double coat of red nail polish was applied leaving only a $4 \mathrm{x} 4 \mathrm{~mm}$ window of enamel exposed in the center of each slab. These 
60 slabs were then allocated into four groups to receive one of the following topical fluoride agents.

Group 1: 38\% SDF (Riva Star, SDI, Bayswater, VIC, Australia)

Group 2: 38\% SDF/KI (Riva Star, SDI, Bayswater, VIC, Australia)

Group 3: $5 \%$ NaF (Colgate ${ }^{\circledR}$ Duraphat ${ }^{\circledR}$ Varnish Single Dose, Colgate Palmolive, Sydney, NSW, Australia)

Group 4: 0.32\% Sodium Fluoride toothpaste (Colgate Total®, Colgate Palmolive, Sydney, NSW, Australia).

Stratified random sampling was used to ensure that SDF was applied to one section while SDF+KI was applied to the second section of the same tooth. NaF and FTP acted as reference and control groups and similarly NaF was applied to one section while FTP was applied to the second section the same tooth to reduce sample bias. Each slab was weighed pre- and post-application of the fluoride agent using an analytical balance to an accuracy of $\pm 0.1 \mathrm{mg}$ (Sartorius, Gottingen, Germany) such that the initial amount of fluoride and subsequent proportion of fluoride released could be analysed. The results of a pilot study revealed $30 \%$ difference in mean fluoride leachate with a standard deviation of $10 \%$ to be clinically relevant. Therefore, with a level of significance set at 0.05 and a power $(1-\beta)$ of 0.85 , five samples per sub-group would be both feasible and sufficient. This is consistent with previous studies investigating fluoride leachate [10-14].

\section{Artificial saliva composition}

Following fluoride application five samples from each group were immersed into $25 \mathrm{ml}$ of either neutral, basic or acidic artificial saliva solutions, as shown in Figure 1. The artificial saliva contained (mmoles/L): $\mathrm{CaCl}_{2}$ (0.7), $\mathrm{MgCl}_{2} \cdot 6 \mathrm{H}_{2} \mathrm{O}(0.2), \mathrm{KH}_{2} \mathrm{PO}_{4}$ (4.0), $\mathrm{KCl}(30)$, $\mathrm{NaN}_{3}$ (0.3), and HEPES buffer (20) as described by Pashley et al. [15]. The neutral solution was stabilized to $\mathrm{pH} 7.0$, the acidic solution buffered to pH4.4 using a lactate buffer and the basic solution buffered to pH10.4 using a glycine/sodium hydroxide buffer. After 24hours each enamel slab was removed from the artificial saliva and gently immersed into a test tube containing $25 \mathrm{ml}$ of new artificial saliva of matched $\mathrm{pH}$. This process was repeated at 48, 96 , and 168hours post-application. The concentration of fluoride in each sample of artificial saliva was measured to determine the amount of fluoride released from the enamel block.

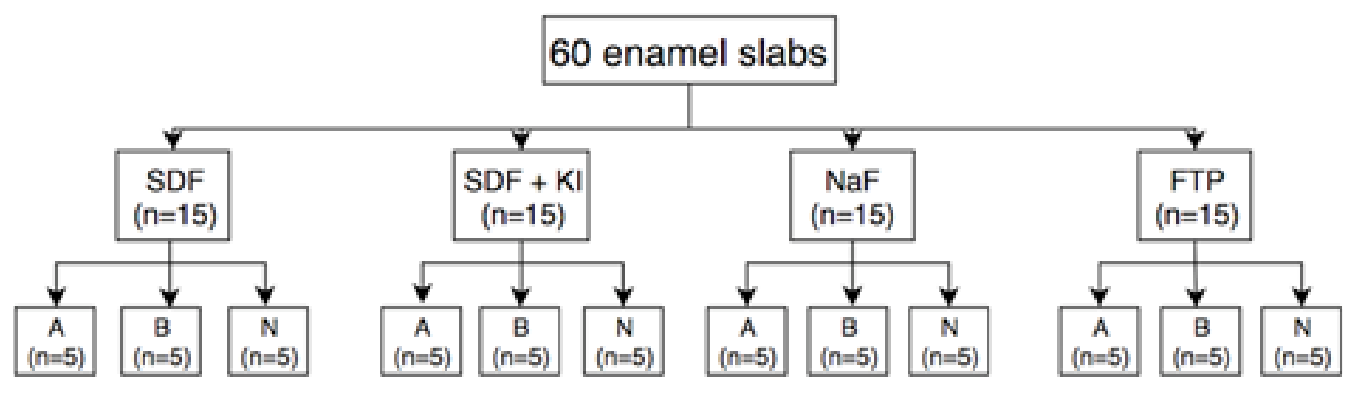

Figure 1: Sample preparation and stratification: 60 enamel slabs were prepared, these were grouped to receive either Silver Diamine Fluoride (SDF), Sodium Fluoride varnish (NaF), Silver Diamine Fluoride followed by Potassium Iodide (SDF+KI) or fluoridated toothpaste (FTP) These groups were subcategorized and the slabs were placed into either acidic, basic or neutral saliva. Fluoride leachate from each of these subgroups was measured at $24,48,96$, and 168 hours post-application.

\section{Fluoride analysis}

Free fluoride ion concentration was measured using a fluoride ion selective electrode (Orion Fluoride Electrode 9609BN, Thermo Electron, Beverly, Massachusetts). The fluoride electrode was calibrated by preparing $100 \mathrm{ml}$ reference solutions containing known concentrations of $1000,100,10,1$, and 0.1 parts per million (ppm) fluoride. The reference solutions were prepared from a $1000 \mathrm{ppm}$ standard fluoride ion solution and deionized water for fluoride uniformity. The fluoride-specific ion electrode was then connected to a digital $\mathrm{pH} / \mathrm{mV}$ meter (model 801A, Orion Research, Cambridge, Massachusetts) and a calibration curve was created by plotting the fluoride concentration (log axis) of the reference solutions against the measured millivoltage. New calibration curves were made using fresh reference solutions each day the measurements were taken. At each time point (i.e. 24, 48, 96, and 168 hours), $7.5 \mathrm{ml}$ of artificial saliva was collected for analysis. This was added to an equal volume of total ionic strength adjusting buffer (TISAB). The TISAB buffer equalized the ionic strength of the solution and acted as a de-complexing agent to release any fluoride ions that were bound up in complex molecules. Three measurements were performed on each sample and the average voltage value was recorded. The values were converted into ppm using the reference calibration curves. Subsequently, these were further converted into micromole per liter of fluoride ions to allow uniformity in analysis.

\section{Data analysis}

One-way analysis of variance (ANOVA) was used to investigate difference across the groups. Post-hoc Tukey's Honestly Significant Difference (HSD) was subsequently used to evaluate differences in the leachate profiles between the groups. The pre- and postapplication weights were used to calculate the baseline fluoride content and the cumulative fluoride release data for each agent as a percentage. 


\section{Result}

\section{Fluoride leachate profiles}

Figure 2 shows the leachate profiles of the various fluoride modalities after 24 and 48hours. At 24hours NaF exhibited the highest fluoride leachate concentration across neutral, acidic and basic groups, this was statistically significant when compared with the other fluoride agents $(\mathrm{p}<0.01)$. At the same time point, SDF showed a higher fluoride leachate than SDF+KI, however this did not reach statistically significance. After 48hours NaF continued to exhibit a higher fluoride leachate in the neutral artificial saliva compared with the other groups $(\mathrm{p}<0.01)$. Although fluoride samples were also taken and measured at 96 and 168 hours postapplication, the concentrations were found to be below $5 \mu \mathrm{mol} / \mathrm{L}$ across all groups and below the sensitivity threshold of the fluoride probe. When evaluating the difference in fluoride release among differing $\mathrm{pH}$, a general trend was observed at 24 hours showing $\mathrm{NaF}$ to exhibit the highest fluoride leachate followed by SDF, SDF+KI and FTP respectively. No significant difference in fluoride leachate was detected between SDF+KI and FTP regardless of the $\mathrm{pH}$.

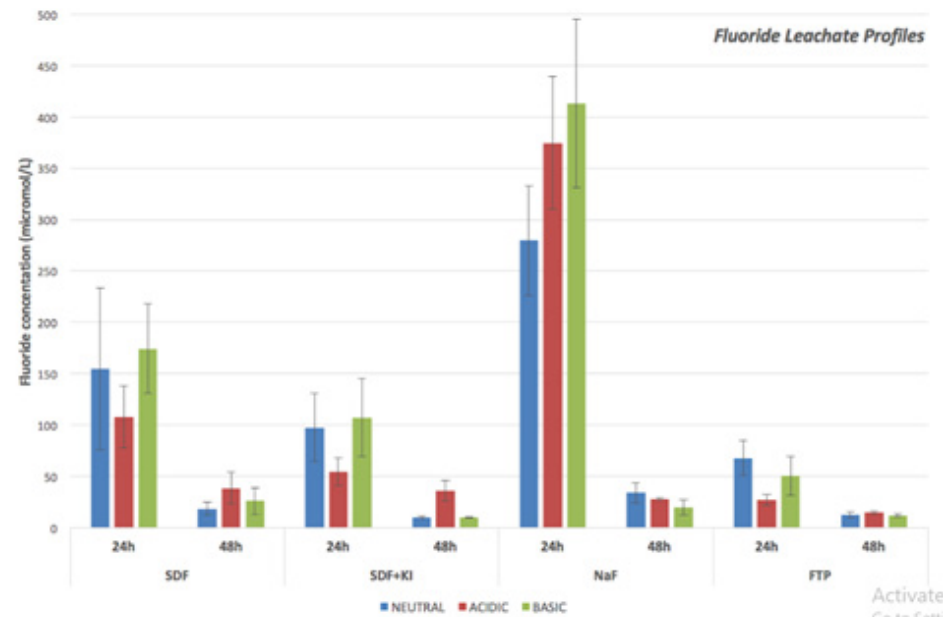

Figure 2: Fluoride leachate profiles of Silver Diamine Fluoride (SDF), Sodium Fluoride varnish (NaF), Silver Diamine Fluoride followed by Potassium Iodide (SDF+KI) and fluoridated toothpaste (FTP) measured at 24 and 48hours post-application.

\section{Cumulative fluoride release}

Figure 3 shows the cumulative fluoride released calculated from the pre-and post-application weights of the various agents. In general, SDF and SDF+KI had a higher proportion of fluoride being released in 24 hours compared to NaF and FTP. This was particularly true in the neutral pH were SDF and SDF+KI had statistically higher cumulative fluoride release when compared to $\mathrm{NaF}(\mathrm{p}<0.05)$. In an acidic environment however, there was no difference between SDF and NaF, but a statistically significant difference was noted between SDF and SDF+KI as well as NaF compared with SDF+KI $(p<0.05)$. Fewer variations were seen in a basic environment with statistically significant differences only occurring between FTP, $\mathrm{SDF}$, and SDF+KI $(\mathrm{p}<0.05)$.

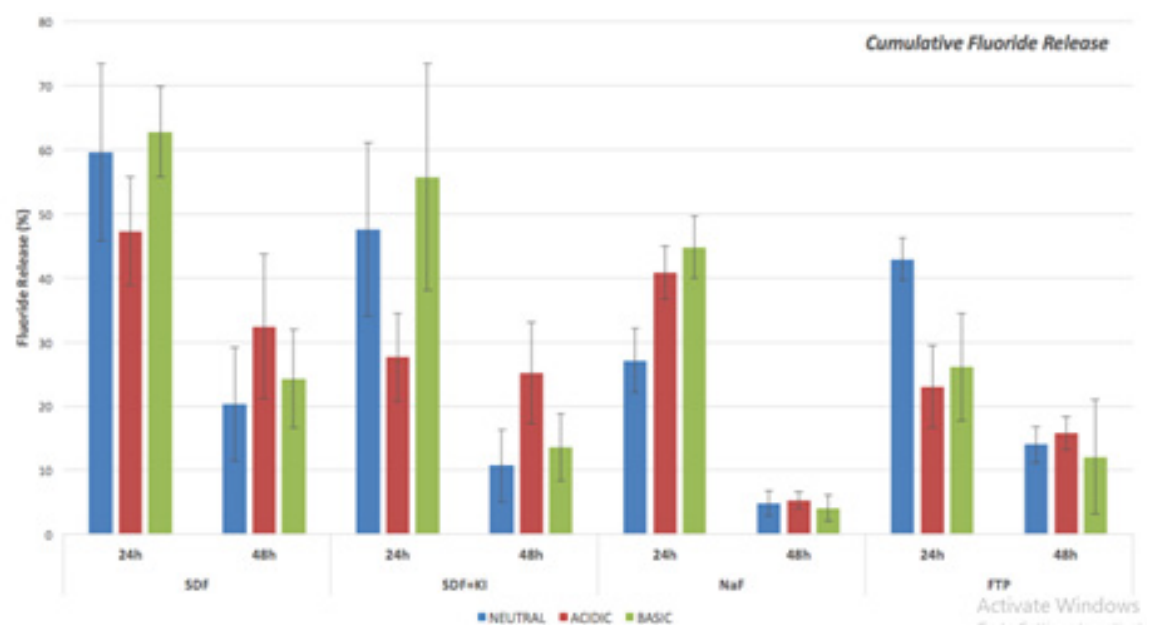

Figure 3: Cumulative fluoride Release of Silver Diamine Fluoride (SDF), Sodium Fluoride varnish (NaF), Silver Diamine Fluoride followed by Potassium Iodide (SDF+KI) and fluoridated toothpaste (FTP) measured at 24 and 48hours post-application. 


\section{Discussion}

To our knowledge, this is the first in vitro study to (i) investigate the fluoride release of SDF as compared with SDF+KI, NaF and FTP, and (ii) explore the fluoride leachate profile of these agents under acidic, neutral and basic conditions. It was interesting to note that $\mathrm{NaF}$ exhibited a higher fluoride leachate compared with SDF, SDF+KI and FTP. Conversely, SDF showed a higher cumulative fluoride release compared to $\mathrm{NaF}$, which may be attributed to the physical properties of the material. The specific interest in SDF centres around the following attributes: established efficacy in arresting carious lesions, ease and simplicity of use, affordability, minimal requirement for specialised training and no significant adverse effects $[1,2,16]$. Previous studies have raised concerns regarding the high fluoride concentrations and subsequent potential toxicity from silver fluoride compounds [6]. It is imperative to exercise caution when interpreting the findings of published studies that have asserted that commercial preparations of silver fluoride may contain unacceptably high levels of fluoride and pose a risk of fluorosis in children $[6,9]$.

These studies investigated silver fluoride rather than SDF; the latter being a much more chemically stable compound $[6,9]$. Furthermore, the preparations may vary in their chemical composition for example, a study investigating silver fluoride found that the commercial preparation actually contained silver difluoride, which contributed to the high fluoride concentrations being measured [8]. The findings of the present study demonstrate that a single application of SDF over a $4 \mathrm{mmx} 4 \mathrm{~mm}$ enamel surface results in an average $0.14 \mathrm{mg}$ of fluoride. This is consistent with the findings of [17] who suggested that the average amount of fluoride following SDF application per patient ranged from approximately $0.17 \mathrm{mg}$ to $0.36 \mathrm{mg}$. From a clinical perspective, the 'No Observed Adverse Effect Limit (NOAEL)' is defined as the highest dose of a chemical in a single study, found by experiment or observation, which causes no detectable adverse health effect [18]. The NOAEL for fluorosis is claimed to be $0.05 \mathrm{mgF} / \mathrm{kg} /$ body weight [18]. The most commonly adopted frequency of SDF application is annually or biannually and restricting SDF application to carious lesions only totals to an amount significantly lower than the NOAEL dose. The fluoride leachate analysis suggests a trend whereby NaF released the highest concentration of fluoride followed by SDF, SDF+KI and FTP groups respectively after 24 hours. This was reflected by posthoc testing which showed a statistically difference between $\mathrm{NaF}$ and the other groups despite of $\mathrm{pH}(\mathrm{p}<0.01)$. The NaF used in this study was a high viscosity varnish that enabled a thicker layer of material to be placed on the enamel slab (mean application weight: $0.01 \mathrm{~g}$ ). Furthermore, the ability of the varnish to tenaciously bind to the tooth structure results in a higher chance of residual $\mathrm{NaF}$ being present on the tooth at follow-up periods as observed in this study. In comparison, SDF is an aqueous solution with a very low film thickness allowing only a small amount to be applied onto the tooth surface (mean application weight: $0.003 \mathrm{~g}$ ). Unlike NaF, SDF is a clear, colourless solution making it is difficult to visualise the true amount applied. The concentration of $\mathrm{NaF}(22,600 \mathrm{ppm})$ is significantly lower than that of SDF $(44,800 \mathrm{ppm})$, yet the fluoride leachate concentration of $\mathrm{NaF}$ was significantly higher than that of SDF.

This can be attributed in part to the minute amount of SDF applied to the enamel surface when compared with $\mathrm{NaF}$. In the oral environment, SDF will be readily diluted by saliva. As the volume of saliva in the oral cavity is estimated to be approximately $0.60 \mathrm{~mL}$ [19], the findings of this study suggest that the concentration of $\mathrm{NaF}$ and SDF per application in the oral cavity to be around $17 \mathrm{mg} /$ $\mathrm{ml}$ and $5 \mathrm{mg} / \mathrm{mL}$ respectively. This illustrates the importance of considering the effective dose applied with respect to saliva and not only the claimed fluoride concentration the topical fluoride agent. The results of cumulative fluoride release exhibited a trend where both SDF and SDF+KI showed higher proportion of fluoride release compared with $\mathrm{NaF}$. This is reflective of the physical characteristics of the agents; $\mathrm{NaF}$ is a varnish in a natural resin base that is not readily soluble, resulting in a lower measurable total soluble fluoride. However, SDF is an aqueous solution that is highly soluble in water and therefore a large amount proportion is leached into the saliva in the first 24 hours. One of the limitations of this study was that the concentrations of samples taken at 96- and 168-hours post-application were found to be below $5 \mu \mathrm{mol} / \mathrm{L}$ across all groups and therefore below the sensitivity threshold of the fluoride ion selective electrode.

However, even fluoride present in low, sustained concentrations (sub-ppm range) is of clinical importance. During demineralization low levels of fluoride in saliva will make the solution highly supersaturated with respect to Fluor hydroxyapatite and thereby enhance remineralization [20]. SDF has shown to have a higher clinical efficacy in arresting carious lesions when compared with other non-surgical approaches [2]. However, the resulting black stain (side effect) of SDF is a concern and affects patients' and/or parents' acceptability of this treatment $[21,22]$. Recently, the use KI has been recommended following SDF application to reduce the degree of black staining [23]. KI can react with free silver ions to produce silver iodide, which is a creamy white reaction product [23]. The results of both the fluoride leachate analysis and cumulative fluoride release show lower fluoride concentrations for SDF+KI compared with SDF alone. This finding may be attributed to the formation of silver iodide, which may physically displace the SDF and therefore the measurable fluoride, when confined to a $4 \mathrm{mmx} 4 \mathrm{~mm}$ enamel slab $[24,25]$. Whether the application of KI leads to a decreased clinical efficacy of SDF as a cariostatic agent is yet to be determined by clinical trials.

\section{Conclusion}

Based on the findings of this study, we conclude that:

(i) NaF exhibited a higher fluoride leachate compared to SDF, $\mathrm{SDF}+\mathrm{KI}$ and TP,

(ii) fluoride leachate profiles varied depending on the $\mathrm{pH}$ of the saliva,

(iii) SDF showed a higher cumulative fluoride release compared to $\mathrm{NaF}$, and 
(iv) $\mathrm{KI}$ application resulted in a lower fluoride release.

\section{Acknowledgement}

The authors would like to thank Mr. Michael Smirk for his assistance and support in the use of the fluoride ion selective electrode.

\section{Conflict of Interest}

The authors declare that they have no conflict of interest

\section{References}

1. Duangthip D, Fung M HT, Wong M, Chu CH, Lo ECM (2017) Adverse effects of silver diamine fluoride treatment among preschool children. J Dent Res 97(4): 395-401.

2. Chibinski AC, Wambier LM, Feltrin J, Loguercio AD, Wambier DS, et al. (2017) Silver diamine fluoride has efficacy in controlling caries progression in primary teeth: A systematic review and meta-analysis. Caries Res 51(5): 527-541.

3. Gao SS, Zhao IS, Hiraishi N, Duangthip D, Mei ML, et al. (2016) Clinical trials of silver diamine fluoride in arresting caries among children: A systematic review. JDR Clin Trans Res 1(3): 201-210.

4. Duangthip D, Chu CH, Lo EC (2016) A randomized clinical trial on arresting dentine caries in preschool children by topical fluorides18month results. J Dent 44: 57-63.

5. Nelson T, Scott JM, Crystal YO, Berg JH, Milgrom P (2016) Silver diamine fluoride in pediatric dentistry training programs: survey of graduate program directors. Pediatr Dent 38(3): 212-217.

6. Gotjamanos T, Afonso F (1997) Unacceptably high levels of fluoride in commercial preparations of silver fluoride. Aust Dent J 42(1): 52-53.

7. Gotjamanos T (1997) Safety issues related to the use of silver fluoride in paediatric dentistry. Aust Dent J 42(3): 166-168.

8. Knight GM, McIntyre JM, Craig GG, Zilm PS, Gully NJ (2005) An in vitro model to measure the effect of a silver fluoride and potassium iodide treatment on the permeability of demineralized dentine to Streptococcus Mutans. Aust Dent J 50(4): 242-245.

9. Mei ML, Chu CH, Lo ECM, Samaranayake LP (2013) Fluoride and silver concentrations of silver diamine fluoride solutions for dental use. Int J Paediatr Dent 23(4): 279-285.

10. Carvalho AS, Cury JA (1999) Fluoride release from some dental materials in different solutions. Oper Dent 24(1): 14-19.
11. Lippert $F$ (2014) Fluoride release from fluoride varnishes under acidic conditions. J Clin Pediatr Dent 39(1): 35-39.

12. Castillo JL, Milgrom P, Kharasch E, Izutsu K, Fey M (2001) Evaluation of fluoride release from commercially available fluoride varnishes. J Am Dent Assoc 132(10): 1389-1392.

13. Jablonowski BL, Bartoloni JA, Hensley DM, Vandewalle KS (2012) Fluoride release from newly marketed fluoride varnishes. Quintessence Int 43(3): 221-228.

14. Castillo JL, Milgrom P (2004) Fluoride release from varnishes in two in vitro protocols. J Am Dent Assoc 135(12): 1696-1699.

15. Pashley DH, Tay FR, Yiu C, Hashimoto M, Breschi L, et al. (2004) Collagen degradation by host-derived enzymes during aging. J Dent Res 83(3): 216-221.

16. Rajendra A, Keenan VA, Oliveira BH, Ruff RR, Wong M, et al. (2017) Topical silver diamine fluoride for managing dental caries in children and adults (Protocol). Cochrane Database Syst Rev 2017(7).

17. Vasquez E, Zegarra G, Chirinos E, Castillo JL, Taves DR, et al. (2012) Short term serum pharmacokinetics of diamine silver fluoride after oral application. BMC Oral Health 12: 60.

18. Browne D, Whelton H, Mullane OD (2005) Fluoride metabolism and fluorosis. J Dent 33(3): 177-186.

19. Lagerlöf F, Dawes C (1984) The volume of saliva in the mouth before and after swallowing. J Dent Res 63(5): 618-621.

20. Buzalaf MA, Pessan JP, Honorio HM, ten Cate JM (2011) Mechanisms of action of fluoride for caries control. Monogr Oral Sci 22: 97-114.

21. Crystal YO, Janal MN, Hamilton DS, Niederman R (2017) Parental perceptions and acceptance of silver diamine fluoride staining. J Am Dent Assoc 148(7): 510-518.

22. Clemens J, Gold J, Chaffin J (2018) Effect and acceptance of silver diamine fluoride treatment on dental caries in primary teeth. J Public Health Dent 78(1): 63-68.

23. Knight GM, McIntyre JM, Mulyani (2006) The effect of silver fluoride and potassium iodide on the bond strength of auto cure glass ionomer cement to dentine. Aust Dent J 51(1): 42-45.

24. Mei ML, Ito L, Cao Y, Lo EC, Li QL, et al. (2014) An ex vivo study of arrested primary teeth caries with silver diamine fluoride therapy. J Dent 42(4): 395-402.

25. Rosenblatt A, Stamford TCM, Niederman R (2009) Silver diamine fluoride: a caries "silver-fluoride bullet". J Dent Res 88(2): 116-125. 